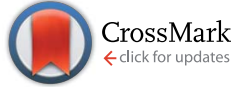

Cite this: RSC Adv., 2016, 6, 93915

Received 11th August 2016

Accepted 26th September 2016

DOI: 10.1039/c6ra20319a

www.rsc.org/advances

\title{
Stretchable and transparent supercapacitors based on aerosol synthesized single-walled carbon nanotube films
}

\author{
Evgenia P. Gilshteyn, ${ }^{a}$ Tanja Kallio, ${ }^{\text {b }}$ Petri Kanninen, ${ }^{\text {b }}$ Ekaterina O. Fedorovskaya, ${ }^{\text {cd }}$ \\ Anton S. Anisimov ${ }^{e}$ and Albert G. Nasibulin*afg
}

Stretchable all-solid supercapacitors based on aerosol synthesized single-walled carbon nanotubes (SWCNTs) have been successfully fabricated and tested. High quality SWCNT films with excellent optoelectrical and mechanical properties were used as the current collectors and active electrodes of the stretchable supercapacitors. A transmittance of up to $75 \%$ was achieved for supercapacitors made from the assembly of two PDMS/SWCNT electrodes and a gel electrolyte in between. The transparent supercapacitor has a specific capacitance of $17.5 \mathrm{~F} \mathrm{~g}^{-1}$ and can be stretched up to $120 \%$ with practically no variation in the electrochemical performance after 1000 stretching cycles and 1000 chargingdischarging cycles.

\section{Introduction}

Transparent energy conversion and storage devices have recently attracted increasing attention due to their great potential as integrated power sources for displays and windows in buildings, automobiles and aerospace vehicles. ${ }^{1-3}$ On the other hand, mechanical stretchability coupled with optical transparency of the energy storage devices is required for many other applications, ranging from self-powered rolled-up displays to self-powered wearable optoelectronics. The design of highly stretchable devices is an essential element in the development of many unprecedented applications such as electronic skin ${ }^{4,5}$ and smart energy storage clothes. ${ }^{6-8}$ Portable and wearable supercapacitors, coupled with either selfhealability or stretchability, ${ }^{7,9,10}$ have particularly become mainstream in personalized electronics. ${ }^{11,12}$ However, the development of both transparent and stretchable supercapacitors (TSSs) is still a challenge, because typical existing

\footnotetext{
${ }^{a}$ Skolkovo Institute of Science and Technology, Laboratory of Nanomaterials, 100 Novaya str., Skolkovo, Moscow Region, 143025, Russia. E-mail: a.nasibulin@ skoltech.ru

${ }^{b}$ Research Group of Electrochemical Energy Conversion and Storage, Department of Chemistry, School of Chemical Technology, Aalto University, P.O. Box 16100, FIo0076 Aalto, Finland. E-mail: tanja.kallio@aalto.fi

${ }^{c}$ Nikolaev Institute of Inorganic Chemistry, Siberian Branch of Russian Academy of Sciences, 3, Acad. Lavrentiev Ave., Novosibirsk, 630090, Russia

${ }^{d}$ Novosibirsk State University, 2 Pirogov str., Novosibirsk, 630090, Russia

${ }^{e}$ Canatu Oy, Konalankuja 5, FI-0039o Helsinki, Finland

${ }^{f}$ Department of Applied Physics, School of Science, Aalto University, P.O. Box 15100, FI00076 Aalto, Finland
}

${ }^{g}$ Saint-Petersburg State Polytechnical University, Department of Material Science, Polytechnicheskaya 29, 195251, Saint-Petersburg, Russia electrodes are neither stretchable nor transparent (e.g., metal oxide on carbon-based electrodes), even though they might possess either stretchability with low transmittance (e.g., conducting polymers) or transparency with poor mechanical properties (e.g., ITO and other metal oxides). To our best knowledge, no optoelectronic nor energy-related devices have been reported to show both good transparency and high stretchability, though many devices with only one function have been reported elsewhere e.g. ref. 13 and 14 .

SWCNTs possess many unique properties, which are advantageous for a wide variety of applications, including stretchable electronics. They have exceptionally high Young's modulus of elasticity and tensile strength and are the strongest known material. ${ }^{15,16}$ The porosity and specific surface area of SWCNT films are very large, and they possess high transparency and flexibility. ${ }^{17}$ In addition, SWCNTs can withstand extremely high currents (up to $10^{9} \mathrm{~A} \mathrm{~cm}^{-2}$ ) making them an ideal replacement for copper and aluminum in fast integrated charge/discharge circuits. SWCNT films are thought to be excellent replacement for traditional transparent electrodes, such as indium-tin oxide (ITO), which suffers from several serious drawbacks including limited flexibility and chemical resistance, rare raw material and high refraction and haze. ${ }^{18}$ SWCNT networks show improved performance and reduced fabrication cost compared to ITO and organic materials that have been studied as low-cost alternatives. ${ }^{19}$ Particularly, aerosol synthesized and collected on a filter SWCNTs can be effectively transferred to any substrate, including stretchable substrate materials such as PDMS (polydimethylsiloxane). They fulfil all the requirements for low-cost, stretchable and transparent electronics..$^{20-22}$ 
Table 1 Literature review of stretchable and/or transparent supercapacitors based on carbon nanotubes

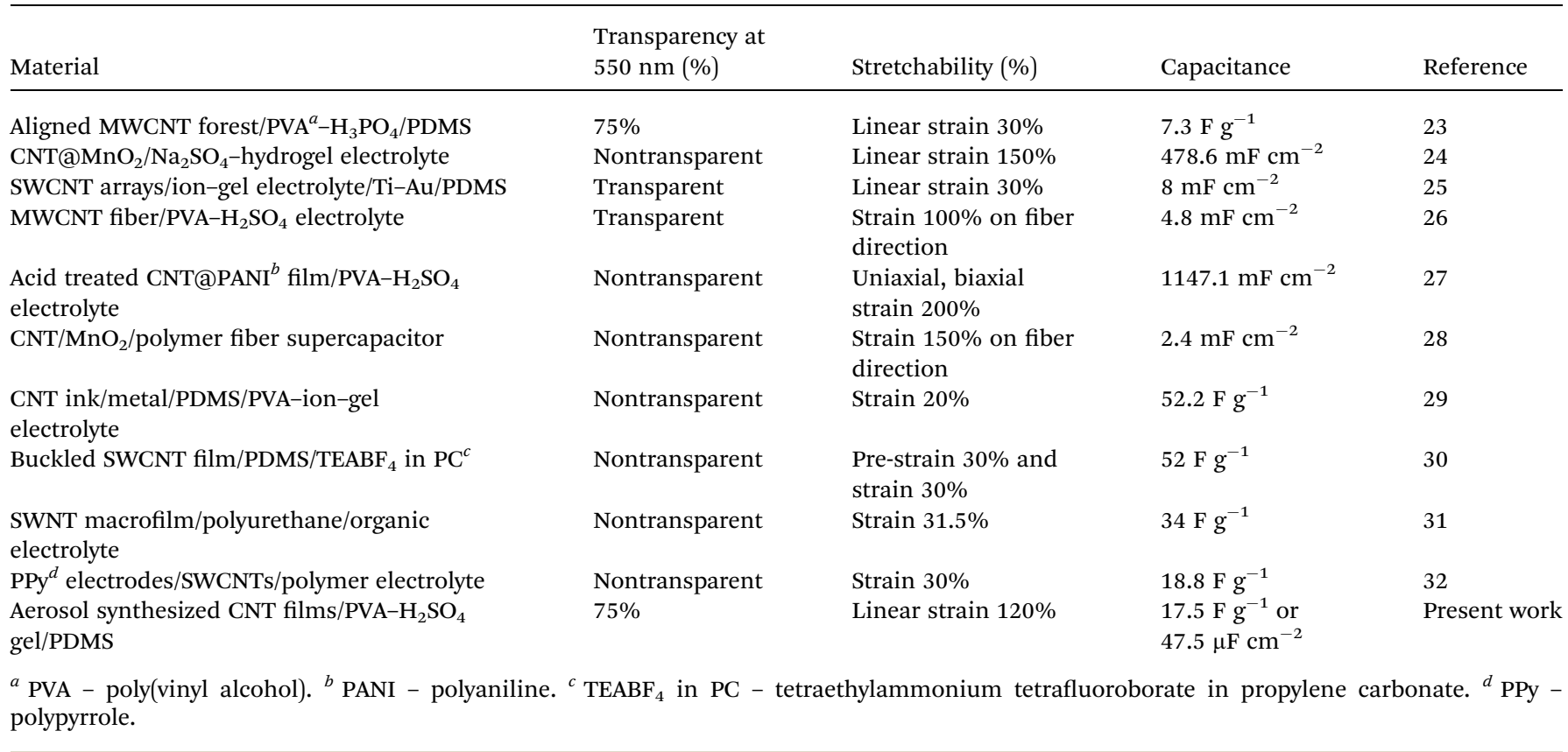

In this work, we applied dry deposited thin films of aerosol CVD synthesized SWCNT as the electrodes to fabricate TSSs. Remarkably high specific capacitance and stability are reached with the SWCNT electrodes in test cells and they are used to construct various TSS prototypes.

Such devices have attracted much attention from researchers and show promising application potential in the field of wearable energy storage. As can be seen from Table 1, where we collected the results devoted to transparent and stretchable supercapacitors based on carbon nanotubes, so far either high transparency or stretchability can be simultaneously achieved in devices. The transparent and stretchable supercapacitors developed in this work, showed a superior specific capacitance of $17.5 \mathrm{~F} \mathrm{~g}^{-1}$ compared to previously reported transparent devices based on CNTs. They can be stretched up to $120 \%$ elongation without any significant change in electrochemical performance under 1000 stretching cycles.

\section{Experimental}

\subsection{Experimental methods and materials}

SWCNTs were synthesized by an aerosol (floating catalyst) CVD method based on ferrocene vapor decomposition in a $\mathrm{CO}$ atmosphere described elsewhere. ${ }^{334}$ Briefly, the catalyst precursor was vaporized by passing ambient pressure and temperature CO through a cartridge filled with a ferrocene powder.

The flow containing ferrocene vapor was then introduced into a high-temperature zone. In order to obtain stable growth of SWCNTs, a controlled amount of carbon dioxide was added together with the carbon source. ${ }^{35}$ SWCNTs were collected downstream of the reactor by filtering the flow through nitrocellulose membrane filters.
SWCNT film morphology was investigated by FEI Helios Nanolab 660 scanning electron microscope with the maximum accelerating voltage of $30 \mathrm{kV}$. TEM images were obtained with a Tecnai G2 F20 transmission electron microscope with a point resolution of $0.24 \mathrm{~nm}$ at $80 \mathrm{kV}$. Raman spectrometer Horiba Jobin-Yvon Labram HR with a $633 \mathrm{~nm}$ laser was used to investigate the quality of the SWCNT film. Optical transmittance was measured with a Perkin Elmer Lambda 1050 UV-Vis-NIR spectrophotometer in a wide range from 150 to $3200 \mathrm{~nm}$, and usually referred to $550 \mathrm{~nm}$.

PDMS substrates with the thickness of about $1 \mathrm{~mm}$ were prepared by pouring the mixture of "base" and "curing" agents at a ratio of $10: 1$ (Sylgard 184, Dow Corning) into a circle molder, followed by thermal curing at $70{ }^{\circ} \mathrm{C}$ for 45 minutes. Then, the PDMS film was cut into required size pieces.

The electrochemical measurements and stability of TSSs was studied with an Autolab PGSTAT100 potentiostat (Metrohm) at various scanning rates. Cyclic voltammograms in the range from -0.8 and $0.8 \mathrm{~V}$ and speed $200 \mathrm{mV} \mathrm{s}^{-1}$ were recorded with an Elins Potentiostat-galvanostat P-40X.

\subsection{Properties of aerosol SWCNT films}

SWCNTs produced by the aerosol CVD method are of a high quality, which can be confirmed with the help of scanning and transmission electron microscope (SEM, TEM) and optical investigations of the produced samples. Thus, the SWCNTs are immediately suitable for many applications without any additional treatment after their deposition on a substrate by a dry press transfer technique. ${ }^{36}$

SEM imaging (Fig. 1a) reveals that the SWCNTs form a random network. This allows to use the SWCNT film as an electrode due to high connectivity resulting in highly conductive 

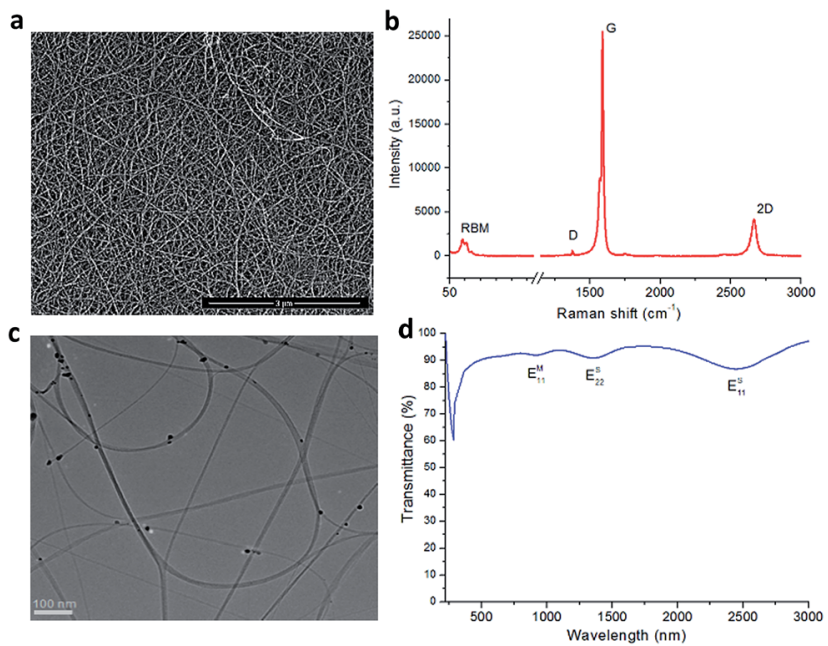

Fig. 1 (a) SEM image of a SWCNT film, (b) Raman spectrum of the SWCNT film with peaks observed: RBM, D, G, 2D, (c) TEM image of the SWCNTs, (d) transmission spectrum of the SWCNT film used for fabrication of TSS electrodes.

surface area readily accessible for ion absorption. Moreover, this kind of an entangled SWCNT network structure allows stretching the electrode so that connections between the mechanically strong SWCNTs remain. Consequently, conductivity is also preserved during stretching and release. TEM images confirmed that the produced tubes are single-walled carbon nanotubes with some iron catalyst nanoparticles (Fig. 1b).

Raman spectrum of the tubes deposited on a quartz substrate demonstrates typical peaks for SWCNTs (Fig. 1c). The D-band around $1350 \mathrm{~cm}^{-1}$ corresponds to defective structures, while the G-band around $1580 \mathrm{~cm}^{-1}$ is indicative of graphitic structures. ${ }^{37}$ The low intensity ratio between $\mathrm{D}$ and $\mathrm{G}$ bands $\left(I_{\mathrm{D}} / I_{\mathrm{G}}=\right.$ 0.029) shows that the structure of the as-produced SWCNTs is highly crystalline and consequently, these nanotubes have good mechanical and electrical properties. In addition, typical Raman peaks for SWCNTs are present: RBM (radial breathing mode), associated with vibration of carbon atoms in a radial direction and $2 \mathrm{D}$ band, the result of two phonon lattice vibrational process.

Van Hove singularities corresponding to electronic transitions in semiconducting $\left(\mathrm{E}_{11}^{\mathrm{S}}\right.$ and $\left.\mathrm{E}_{22}^{\mathrm{S}}\right)$ and metallic $\left(\mathrm{E}_{11}^{\mathrm{M}}\right)$ SWCNTs can be clearly observed in the transmission spectrum (Fig. 1d). The mean diameter of SWCNTs estimated from the spectrum is about $2.5 \mathrm{~nm},{ }^{38}$ which corresponds to the results obtained from the analysis of the TEM images. Optical transmittance of the film is usually referred to the wavelength of $550 \mathrm{~nm}$ (middle of the visible light range - 380 to $780 \mathrm{~nm}$ ) and the quartz substrate contribution is subtracted as the background. Since absorbance, $A$, is a linear function of the SWCNT film thickness, $h$, the latter can be calculated using $h(\mathrm{~nm})=417$ $\times A{ }^{39}$ Therefore, for a film with the transmittance of $90 \%$ the calculated thickness is about $19 \mathrm{~nm}$.

For the TSS fabrication we utilized the films with different transmittances, i.e. thicknesses. SWCNT films with $90 \%$ transmittance were used for fabrication of transparent electrodes ( $\mathrm{CNT}_{1}$ in Fig. 2), while $80 \%$ transmittance ( $40 \mathrm{~nm}$ thick) films

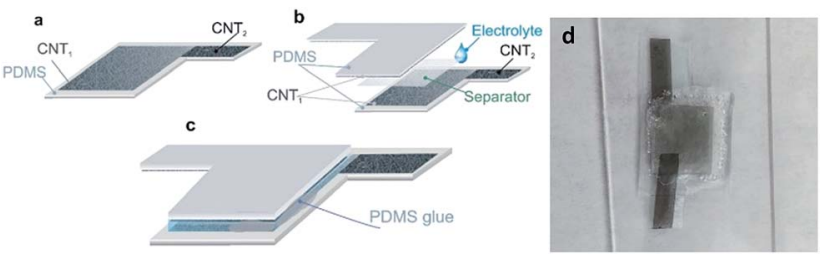

Fig. 2 Scheme of TSS fabrication process: (a) deposition of $\mathrm{CNT}_{1}$ and $\mathrm{CNT}_{2}$ films on PDMS, (b) assembling the TSS by adding the separator and acid electrolyte, (c) gluing two substrates with the liquid PDMS, (d) photograph of the SWCNT based TSS with $\mathrm{H}_{2} \mathrm{SO}_{4}$ electrolyte and a nontransparent separator.

for current collectors $\left(\mathrm{CNT}_{2}\right.$ in Fig. 2), due to the higher conductivity of the thicker SWCNT network.

\section{Results}

\subsection{Fabrication of TSS based on liquid $\mathrm{H}_{2} \mathrm{SO}_{4} /$ separator}

Fig. 2 schematically shows the procedure for preparing transparent and stretchable supercapacitors with liquid $\mathrm{H}_{2} \mathrm{SO}_{4} /$ separator. The pristine SWCNTs were first deposited onto a preformed PDMS substrate (Fig. 2a) and then heated to $200^{\circ} \mathrm{C}$ in order to anneal the surface and to desorb oxygen and organic impurities. After that $2 \mathrm{~mol} \mathrm{dm}^{-3} \mathrm{H}_{2} \mathrm{SO}_{4}$ solution was dropped on top of one of the SWCNT films and covered with a paper separator (Fig. 2b). Finally, we constructed the supercapacitors by assembling another PDMS-supported SWCNT electrode on top of the newly formed separator/liquid $\mathrm{H}_{2} \mathrm{SO}_{4} / \mathrm{SWCNT} / \mathrm{PDMS}$ multilayer film and glued this structure with liquid PDMS at the edges. Fig. 2 shows the fabricated stretchable and semitransparent device. The fabricated supercapacitor is flexible as it can be seen from Fig. $2 \mathrm{~d}$ and can be stretched at least up to $50 \%$ strain (Fig. $4 \mathrm{~b}$ ), as will be demonstrated further.

Immediately after the liquid PDMS glue was solidified, cyclic voltammogram characteristics were measured at different scan rates from 10 to $500 \mathrm{mV} \mathrm{s}^{-1}$ (Fig. 3). The current increased with the increasing scan rate, as is characteristic for adsorption-controlled reactions, implying that no mass transfer or kinetic limitations can be observed and all the SWCNT surface sites are readily accessible even with the
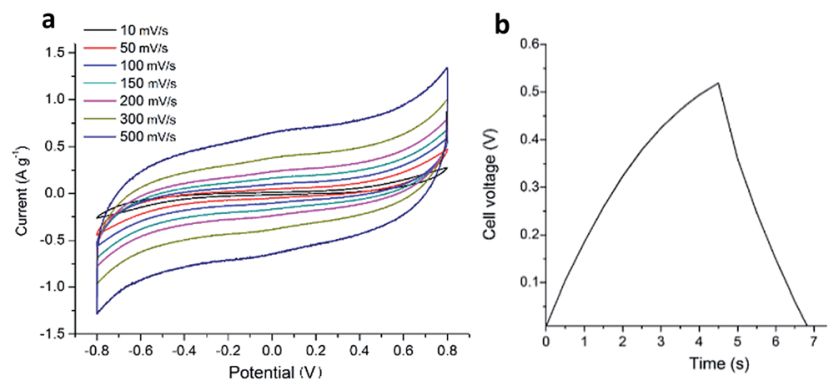

Fig. 3 Electrochemical characterization of the SWCNT stretchable supercapacitors with acid $\mathrm{H}_{2} \mathrm{SO}_{4}$ : (a) cyclic voltammograms at various scan rates (numbers indicates scan rates in $\mathrm{mV} \mathrm{s}^{-1}$ ), (b) constant current charging and discharging at $0.25 \mathrm{~A} \mathrm{~g}^{-1}$. 

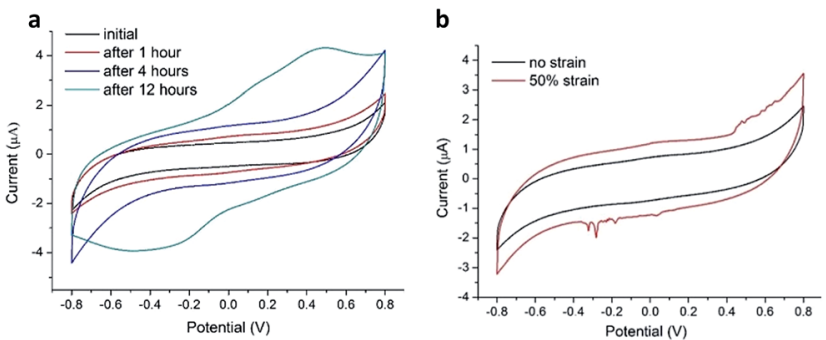

Fig. 4 (a) Cyclic voltammograms of TSS with liquid acidic electrolyte at different periods of time after the fabrication, (b) cyclic voltammograms of TSS before stretching and being stretched at $50 \%$.

higher scan rates. Thus, the same capacitance is achieved in the studied scan rate range.

Due to the fact that we used water based electrolyte and SWCNTs exhibit hydrophobic behavior, cyclic voltammograms measured at different periods of time (Fig. 4a) showed the time dependence. To follow the wetting behavior of the electrodes, the voltammograms were measured immediately after the fabrication process, after 1, 4 and 12 hours. It can be easily seen that after letting the device be impregnated in electrolyte for 12 hours the current increased 6 times.

To analyze the stretchability of the transparent supercapacitors, cyclic voltammetric measurements were performed under mechanical deformations, such as stretching after the fabrication process (Fig. 4b). It was observed that the capacitance of the TSS was improved by stretching up to $50 \%$ strain. This can be explained by the fact that stretching process led to better wetting of the SWCNTs by the electrolyte, thus increasing the area available for the ion absorption.

The specific capacitance of this device $\left(C_{\mathrm{sp}}\right)$ can be calculated using the charge integrated from the area of the cyclic voltammogram graphs according to: ${ }^{30-32}$

$$
C_{\mathrm{sp}}=\frac{1}{2 m V \nu} \int_{V_{-}}^{V_{+}} I(V) \mathrm{d} V
$$

where $m$ is the mass of the active material in both electrodes, $V$ $=0.8 \mathrm{~V}$ is the potential window between the positive and negative electrodes, $\nu=200 \mathrm{mV} \mathrm{s}^{-1}$ is the scan rate. The mass density of the electrodes was determined to be $5 \mu \mathrm{g} \mathrm{cm}{ }^{-2}$. This type of TSSs has demonstrated the capacitance $C_{\mathrm{sp}}$ of $3.2 \mathrm{~F} \mathrm{~g}^{-1}$, and can be taken as a basis for the comparison with the results obtained with improved structures.

One of the main issues impeded the fabrication of highly stretchable supercapacitors with high performance is the leakage of the electrolyte while applying force to the TSSs.

This problem is arisen from the configuration of the stretchable supercapacitor device wherein liquid electrolyte is sandwiched by two electrodes. Our further experiments were devoted to improvement of TSS configuration by replacing the liquid electrolyte, which might suffer from leakage of a harmful liquid, and consequently the non-transparent separator with a conformable gel electrolyte.

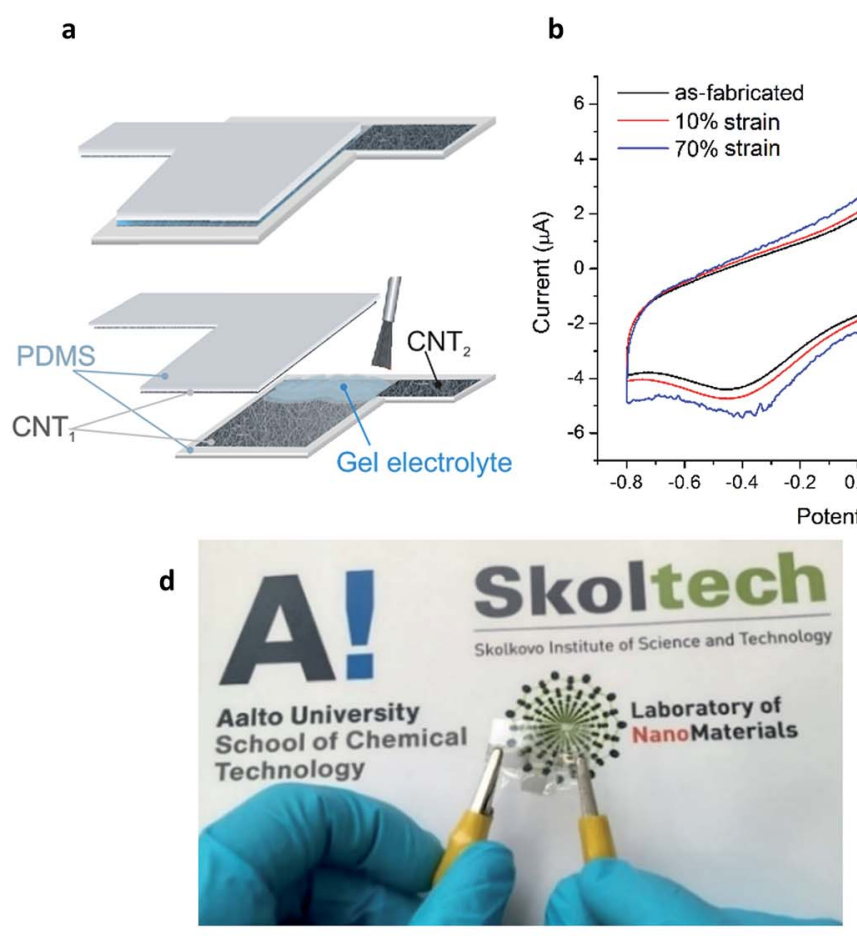

c 


\subsection{Fabrication of TSS based on a $\mathrm{PVA}-\mathrm{H}_{2} \mathrm{SO}_{4}$ gel electrolyte}

Nonetheless, most reports on stretchable supercapacitors have focused on the development of stretchable electrode materials and their designs while little attention is paid on the choice of an electrolyte. Widely used electrolytes are generally liquid. However, the liquid electrolytes are not appropriate for stretchable supercapacitors because of the intrinsic leakage problem. In order to improve the TSS performance we utilized another type of an electrolyte, polyvinyl alcohol- $\mathrm{H}_{2} \mathrm{SO}_{4}$ gel.

The first two fabrication steps were similar to the ones described in the previously reported section (Fig. 2a and b). A gel containing polyvinyl alcohol (PVA) powder $(5 \mathrm{~g})$ and $\mathrm{H}_{2} \mathrm{SO}_{4}(5$ $\mathrm{g}, 95 \%$ concentration) in water $(50 \mathrm{~mL})$ was used as the electrolyte. It is worth nothing that the strength of the $\mathrm{H}_{2} \mathrm{SO}_{4}$ was $95 \%$. Then, the viscous solutions were cast onto the SWCNT electrodes, subsequently the two electrodes coated with the gel electrolyte were dried in the air at room temperature for $10 \mathrm{~h}$. While the solution was still viscous, one of the electrolytecoated electrodes was firmly pressed on the other electrolytecoated electrode and the whole assembly was treated at $40{ }^{\circ} \mathrm{C}$ for $5 \mathrm{~h}$, while the whole supercapacitor became solidified but still fully stretchable (Fig. 5a). Finally, the TSS contacting pads were connected to the potentiostat-galvanostat for subsequent characterization of the device performance (Fig. 5b and c). The whole device can be bent, folded, and even stretched at least up to $70 \%$ strain without any obvious performance change, as it can be seen from Fig. 5b. Additional advantages of this supercapacitor is its high transparency $-75 \%$ (Fig. 5e) and absence of harmful liquid compounds, since the TSS is solid.

\section{Discussions}

The current values can be compared with the values obtained with the acid liquid electrolyte, but the shape of this graph looks better, which indicates better operating parameters of such TSSs. This can be attributed to excellent properties of the PVA$\mathrm{H}_{2} \mathrm{SO}_{4}$ electrolyte, less overall resistance because of a thinner electrolyte layer and high tensile strength after solidification, in comparison with TSS based on the liquid electrolyte. ${ }^{34}$ The capacitance of the device, $C_{\mathrm{sp}}$, calculated according to eqn (1) achieves the value of $7.4 \mathrm{~F} \mathrm{~g}^{-1}$.

Thus, the problems in fabrication of highly stretchable supercapacitors, leakage of the electrolyte, was solved by using the gel electrolyte. For further improvement of TSS characteristics under applied strain we developed pre-stretching approach (Fig. 6a), which is based on spreading of gel electrolyte on the SWCNT film under applied strain. We achieved TSS structure, which can be stretched up to $120 \%$ of strain without significant change of the capacitance even after 1000 stretching cycles (Fig. 6b). The gel electrolyte is viscous and thus stretching improves the contact between the SWCNT electrodes and the electrolyte. Better wetting of the SWCNTs increases the active surface area and consequently, enhances mass transfer and double layer structuring resulting in the observed higher specific capacitance. In Fig. $6 c$ the cyclic voltammograms of the TSS before and after 1000 cycles between -0.8 and $0.8 \mathrm{~V}$ are shown and surprisingly, a small improvement in current is seen. This is attributed to better wetting of the hydrophobic SWCNT during the successive potential cycles and indicates excellent stability for the SWCNT electrodes and the TSS device.

For this pre-stretching approach, the calculated specific capacitance value of $C_{\mathrm{sp}}$ is $17.5 \mathrm{~F} \mathrm{~g}^{-1}$, which is higher than that obtained for the liquid or gel electrolyte without pre-stretching. It ought to be remarked that recently we have fabricated transparent and flexible electrochemical double-layer capacitor (EDLC) prototype from our SWCNT films. ${ }^{17}$ The supercapacitor showed extremely high mass specific capacitance of $178 \mathrm{~F} \mathrm{~g}^{-1}$ compared to other carbon-based flexible and transparent EDLCs. However, that supercapacitor had higher values of the capacitance due to the fact that material properties were tested in a test cell, and the lateral conductivity of the SWCNT film was not an issue. Therefore, the TSS fabricated here demonstrated relatively high capacitance in a prototype cell being stretchable and transparent. Thus, we optimized and eventually fabricated highly stretchable TSS by combining our SWCNT films pre- a

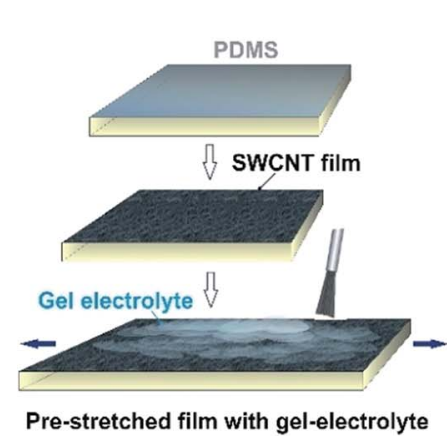

b

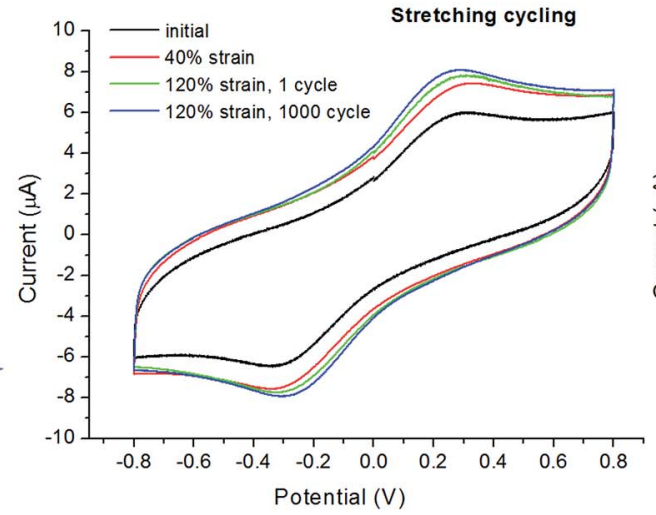

C

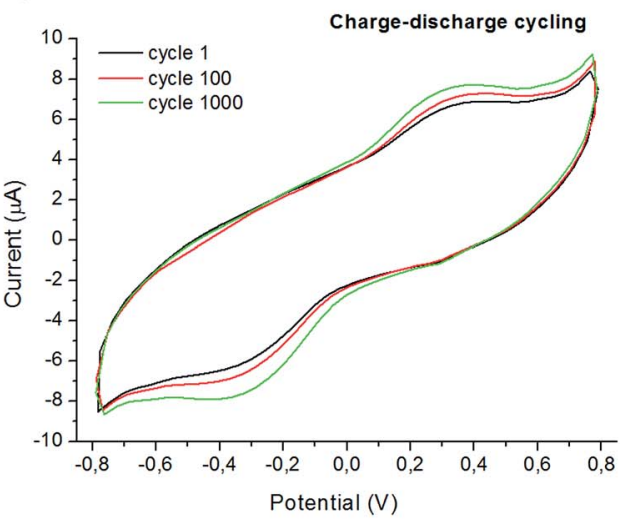

Fig. 6 (a) Scheme of gel electrolyte deposition on pre-stretched SWCNTs on PDMS. (b) Cyclic voltammogramms of TSS made of pre-stretched electrodes at zero applied strain, $40 \%$ strain, at first cycle of stretching to $120 \%$ and after 1000 cycle of stretching to $120 \%$ (scan rate $-200 \mathrm{mV}$ $\mathrm{s}^{-1}$ ), (c) cyclic voltammogram of the supercapacitor before and after 1000 cycles between -0.8 and $0.8 \mathrm{~V}$. 
strained on the PDMS substrate and using of a gel electrolyte. The capacitance of these pre-strained TSSs remained nearly unchanged, even under the strain up to $120 \%$. This configuration enabled stretching of the supercapacitor as an integrated unit and overcame the limitation of conventional stretchable supercapacitor configuration wherein two electrodes move relative to the separator under strain. The performance of the stretchable supercapacitors remained nearly unchanged under $120 \%$ strain even under stretched conditions and after 1000 stretching cycles. Superior stretchability of the aerosol synthesized SWCNT films may find broad applications in stretchable electronics, energy storage electrodes and sensors. Such high stretchability, combined with transparency and high specific capacitance confirmed that fabricated supercapacitor has a great potential for broad practical applications.

\section{Conclusions}

In summary, we successfully developed highly stretchable supercapacitors, based on SWCNT films with $80 \%$ transmittance as current collectors and $90 \%$ transmittance films, as electrode material. Our first fabricated and tested TSSs with liquid $2 \mathrm{~mol} \mathrm{dm}^{-3} \mathrm{H}_{2} \mathrm{SO}_{4}$ electrolyte showed capacitance about $3.2 \mathrm{~F} \mathrm{~g}^{-1}$ and allowed to apply $50 \%$ of strain. This limitation can be explained by the presence of non-stretchable separator and leakage of liquid electrolyte. In order to solve this problem, TSS based on PVA- $\mathrm{H}_{2} \mathrm{SO}_{4}$ gel electrolyte was developed, which showed specific cell capacitance of $7.4 \mathrm{~F} \mathrm{~g}^{-1}$. In order to increase durability to stretching of such TSS another technique was performed based on pre-stretching of the electrodes with the deposited gel electrolyte. This approach allowed using the effect of better electrolyte diffusion and electron transport through the electrode layers and obtain the capacitance value of 17.5 $\mathrm{F} \mathrm{g}^{-1}$, significantly higher than previously reported. Furthermore, TSS based on pre-stretching approach demonstrated stretchability up to $120 \%$ with excellent stability even after stretching for 1000 cycles in addition to electrochemical stability after 1000 charging-discharging cycles.

\section{Acknowledgements}

This work was supported by Skoltech NGP Program (SkoltechMIT joint project).

\section{Notes and references}

1 J. S. Huang, G. Li and Y. Yang, A semi-transparent plastic solar cell fabricated by a lamination process, Adv. Mater., 2008, 20, 415-419.

2 C.-C. Chen, et al., Visibly transparent polymer solar cells produced by solution processing, ACS Nano, 2012, 6, 71857190.

3 H. Y. Jung, M. B. Karimi, M. G. Hahm, P. M. Ajayan and Y. J. Jung, Transparent, flexible supercapacitors from nanoengineered carbon films, Sci. Rep., 2012, 2, 773-777.

4 K. Takei, T. Takahashi, J. C. Ho, H. Ko, A. G. Gillies, P. W. Leu, R. S. Fearing and A. Javey, Carbon nanotube active-matrix backplanes for conformal electronics and sensors, Nat. Mater., 2010, 9, 821-826.

5 S. C. B. Mannsfeld, B. C. K. Tee, R. M. Stoltenberg, C. V. H. H. Chen, S. Barman, B. V. O. Muir, A. N. Sokolov, C. Reese and Z. N. Bao, Highly sensitive flexible pressure sensors with microstructured rubber dielectric layers, Nat. Mater., 2010, 9, 859-864.

6 D.-H. Kim, N. Lu, R. Ma, Y.-S. Kim, R.-H. Kim, S. Wang, J. Wu, S. M. Won, H. Tao, A. Islam, K. J. Yu, T.-i. Kim, R. Chowdhury, M. Ying, L. Xu, M. Li, H.-J. Chung, H. Keum, M. McCormick, P. Liu, Y.-W. Zhang, F. G. Omenetto, Y. Huang, T. Coleman and J. A. Rogers, Epidermal Electronics, Science, 2011, 333, 838-843.

7 L. Hu, M. Pasta, F. L. Mantia, L. Cui, S. Jeong, H. D. Deshazer, J. W. Choi, S. M. Han and Y. Cui, Aqueous Supercapacitors on Conductive Cotton, Nano Lett., 2010, 10, 708-714.

8 B. Yue, C. Wang, X. Ding and G. G. Wallace, Polypyrrole coated nylon lycra fabric as stretchable electrode for supercapacitor applications, Electrochim. Acta, 2012, 68, 1824.

9 C. Yu, C. Masarapu, J. Rong, B. Wei and H. Jiang, Stretchable Supercapacitors Based on Buckled Single-Walled CarbonNanotube Macrofilms, Adv. Mater., 2009, 21, 4793-4797.

$10 \mathrm{X}$. Li, T. Gu and B. Wei, Dynamic and galvanic stability of stretchable supercapacitors, Nano Lett., 2012, 12, 6366-6371.

11 M. Winter and R. J. Brodd, What are batteries, fuel cells, and supercapacitors?, Chem. Rev., 2004, 104, 4245-4270.

$12 \mathrm{~J}$. B. Kim, et al., Wrinkles and deep folds as photonic structures in photovoltaics., Nat. Photonics, 2012, 6, 327-332.

$13 \mathrm{Z}$. Yu, X. Niu, Z. Liu and Q. Pei, Intrinsically stretchable polymer light-emitting devices using carbon nanotubepolymer composite electrodes, Adv. Mater., 2011, 23, 39893994.

14 H. Lin, L. Li, J. Ren, Z. Cai, L. Qiu, Z. Yang and H. Peng, Conducting polymer composite film incorporated with aligned carbon nanotubes for transparent, flexible and efficient supercapacitor, Sci. Rep., 2012, 3, 1353-1358.

15 M. Yu, B. S. Files, S. Arepalli and R. S. Ruoff, Tensile Loading of Ropes of Single Wall Carbon Nanotubes and their Mechanical Properties, Phys. Rev. Lett., 2000, 84, 5552-5555.

16 A. K. Lau and D. Hui, The revolutionary creation of new advanced materials-carbon nanotube composites, Composites, Part B, 2002, 33, 263-277.

17 P. Kanninen, N. D. Luong, L. H. Sinh, I. V. Anoshkin, A. Tsapenko, J. Seppälä, A. G. Nasibulin and T. Kallio, Transparent and flexible high-performance supercapacitors based on single-walled carbon nanotube films, Nanotechnology, 2016, 27, 235403.

18 A. Kaskela, A. G. Nasibulin, M. Zavodchikova, B. Aitchison, A. Papadimitratos, Y. Tian, Z. Zhu, H. Jiang, D. P. Brown, A. Zakhidov and E. I. Kauppinen, Nano Lett., 2010, 10, 4349-4355.

19 Z. Wu, Z. Chen, X. Du, J. M. Logan, J. Sippel and M. Nikolou, et.al., Transparent, Conductive Carbon Nanotube Films, Science, 2004, 27, 1273-1276. 
20 D.-M. Sun, M. Y. Timmermans, A. Kaskela, A. G. Nasibulin, S. Kishimoto, T. Mizutani, E. I. Kauppinen and Y. Ohno, Nat. Commun., 2013, 4, 2302.

21 F. Sajed and C. Rutherglen, All-printed and transparent single walled carbon nanotube thin film transistor devices, Appl. Phys. Lett., 2013, 103, 143303.

22 L. Nyholm, G. Nystrom, A. Mihranyan and M. Stromme, Toward Flexible Polymer and Paper-Based Energy Storage Devices, Adv. Mater., 2011, 23, 3751-3769.

23 T. Chen, H. Peng, M. Durstock and L. Dai, High-performance transparent and stretchable all-solid supercapacitors based on highly aligned carbon nanotube sheets, Sci. Rep., 2014, 4, 3612 .

24 Q. Tang, M. Chen, G. Wang, H. Bao and P. Saha, A facile prestrain-stick-release assembly of stretchable supercapacitors based on highly stretchable and sticky hydrogel electrolyte, J. Power Sources, 2015, 284, 400-408.

25 D. Kim, G. Shin, Y. J. Kang, W. Kim and J. S. Ha, Fabrication of a Stretchable Solid-State Micro-Supercapacitor Array, ACS Nano, 2013, 7, 7975.

26 P. Xu, T. Gu, Z. Cao, B. Wei, J. Yu and F. Li, et.al., Carbon Nanotube Fiber Based Stretchable Wire-Shaped Supercapacitors, Adv. Energy Mater., 2014, 4, 1300759.

$27 \mathrm{~J} . \mathrm{Yu}, \mathrm{W} . \mathrm{Lu}, \mathrm{S}$. Pei, K. Gong, L. Wang and L. Meng, et.al., Omnidirectionally Stretchable High-Performance Supercapacitor Based on Isotropic Buckled Carbon Nanotube Films, ACS Nano, 2016, 10, 5204-5211.

28 C. Choi, S. H. Kim, H. J. Sim, J. A. Lee and A. Young Choi, et.al., Stretchable, Weavable Coiled Carbon Nanotube/ $\mathrm{MnO}_{2} /$ Polymer Fiber Solid-State Supercapacitors, Sci. Rep., 2015, 5, 938.

29 C. Yu, C. Masarapu, J. Rong, B. Wei and H. Jiang, Stretchable Supercapacitors Based on Buckled Single-Walled CarbonNanotube Macro-Films, Adv. Mater., 2009, 21, 4793-4797.

$30 \mathrm{~J}$. Lee, W. Kim and W. Kim, Stretchable Carbon Nanotube/ Ion-Gel Supercapacitors with High Durability Realized through Interfacial Microroughness, ACS Appl. Mater. Interfaces, 2014, 6, 13578-13586.
$31 \mathrm{X}$. Li, T. Gu and B. Wei, Dynamic and Galvanic Stability of Stretchable Supercapacitors, Nano Lett., 2012, 12, 63666371.

32 C. Zhao, C. Wang, Z. Yue, K. Shu and G. G. Wallace, Intrinsically Stretchable Supercapacitors Composed of Polypyrrole Electrodes and Highly Stretchable Gel Electrolyte, ACS Appl. Mater. Interfaces, 2013, 5, 9008-9014.

33 A. Moisala, A. G. Nasibulin, D. P. Brown, H. Jiang, L. Khriachtchev and E. I. Kauppinen, Single-walled carbon nanotube synthesis using ferrocene and iron pentacarbonyl in a laminar flow reactor, Chem. Eng. Sci., 2006, 61, 4393-4402.

34 Y. Tian, M. Timmermans, S. Kivistö, A. Nasibulin, Z. Zhu, H. Jiang, O. Okhotnikov and E. Kauppinen, Tailoring the diameter of single-walled carbon nanotubes for optical applications, Nano Res., 2011, 4, 807-815.

35 A. G. Nasibulin, D. P. Brown, P. Queipo, D. Gonzalez, H. Jiang and E. I. Kauppinen, An essential role of $\mathrm{CO}_{2}$ and $\mathrm{H}_{2} \mathrm{O}$ during single-walled CNT synthesis from carbon monoxide, Chem. Phys. Lett., 2006, 417, 179-184.

36 K. Mustonen, T. Susi, A. Kaskela, P. Laiho, Y. Tian, A. G. Nasibulin and E. I. Kauppinen, Influence of the diameter of single-walled carbon nanotube bundles on the optoelectronic performance of dry-deposited thin films, Beilstein J. Nanotechnol., 2012, 3, 692-702.

37 M. S. Dresselhaus, G. Dresselhaus, R. Saito and A. Jorio, Raman spectroscopy of carbon nanotubes, Phys. Rep., 2005, 409, 47-99.

38 M. S. Dresselhaus, D. Dresselhaus, A. Jorio, et al., Raman spectroscopy on isolated single wall carbon nanotubes, Carbon, 2002, 40, 2043-2061.

39 A. L. Gorkina, A. P. Tsapenko, E. P. Gilshteyn, T. S. Koltsova, T. V. Larionova, A. Talyzin, A. S. Anisimov, I. V. Anoshkin, E. I. Kauppinen, O. V. Tolochko and A. G. Nasibulin, Transparent and Conductive Hybrid Graphene/Carbon Nanotube Films, Carbon, 2016, 100, 501-507. 